\title{
The surface adsorption for Be and Be/00Cr17Ni14Mo2 stainless steel welding joint by diffusion bonding
}

\author{
Hui $\mathrm{Li}^{1, \text { a }}$, Peng-cheng Zhang ${ }^{2}$, Xuan Wang ${ }^{1, b}$, Yong Zhu ${ }^{1, c}$ \\ ${ }^{1}$ Chongqing Academy of Metrology and Quality Inspection, Chongqing 401123, China \\ ${ }^{2}$ National Key Laboratory for Surface Physics and Chemistry, Mianyang 621907, China \\ alihui@cqu.edu.cn, ${ }^{\mathrm{b}} 13667669535 @ 163 . c o m,{ }^{\mathrm{c}} 13883583338 @ 163 . c 0 m$
}

Keywords: adsorption; oxidation; diffusion bonding; Beryllium; 00Cr17Ni14Mo2 stainless steel

\begin{abstract}
Beryllium is a specific metal, its atomic configuration is $1 s^{1} 2 s^{2}$ and affects the shape of $s$ valence band and p valence band because there is no electron in the p sublayer, which dimers bond length is $11 \%$ more than inside and (0001) atomic spacing shows $6 \%$ abnormal expansion. Compared with other elements, beryllium shows abnormal surface properties which the surface adsorption energy is up to $3.32 \mathrm{eV} /$ atom due to electron exciting from s layer to p layer in the solid. In this paper, the adsorption and oxidation properties for Be and Be/00Cr17Ni14Mo2 stainless steel bonding joint were investigated by auger electron spectroscopy (AES) and two secondary ion mass spectrometry (SIMS). The results show that Beryllium, welding seam and 00Cr17Ni14Mo2 stainless steel matrix with beryllium adsorbed strongly in $\mathrm{C}$ and $\mathrm{O}$ then seriously oxidized even in super high-vacuum atmosphere, whose compounds remained in the welding joint and formed inclusion and segregation, and resulted in reducing bonding strength. Thus, pretreatment of beryllium surface and control of bonding atmosphere are significantly important, which can determine directly the success or failure of diffusion bonding for Be/00Cr17Ni14Mo2 stainless steel, and provides a guide for beryllium welding with other material.
\end{abstract}

\section{Introduction}

Beryllium is a reactor material because it has the lowest $Z$, relatively high thermal conductivity, and low activation, etc. Diffusion bonding is an effective way to join beryllium with other materials. With this process, joining of materials is achieved through atomic transport and mechanical processes at the bonding interface. In recent years, scholars carried on a lot of research on the adsorption, oxidation and corrosion of beryllium[1]-[11], which show that there is oxide film in the beryllium surface and seriously effects on improving bonding strength. In this paper, the adsorption and oxidation properties for $\mathrm{Be}$ and $\mathrm{Be} / 00 \mathrm{Cr} 17 \mathrm{Ni} 14 \mathrm{Mo} 2$ stainless steel bonding joint were investigated by auger electron spectroscopy (AES) and two secondary ion mass spectrometry (SIMS). It was found that beryllium adsorbed strongly in $\mathrm{C}$ and $\mathrm{O}$ then seriously oxidized even in super high-vacuum atmosphere, whose compounds were brittle, left no continuous void and micro crack in the welding joint, and resulted in decreasing bonding strength. Thus, pretreatment of beryllium surface and control of bonding atmosphere are significantly important, and there is realistic significance for beryllium welding with other material.

\section{Experiments}

\section{Materials and Specimens}

Beryllium specimens are made of hot-pressed beryllium, which contains $0.61 \% \mathrm{BeO}, 0.027 \%$ Be2C and $0.08 \%$ Fe. 00Cr17Ni14Mo2 stainless steel (SS) is the austenite anti- hydrogen stainless steel, which contains $17.2 \% \mathrm{Cr}, 14.27 \% \mathrm{Ni}, 2.19 \% \mathrm{Mo}$ and $1.05 \% \mathrm{Mn}$, and its impurity total contents is less than $0.17 \%$. Bars of Be and SS are $12 \mathrm{~mm}$ in diameter and $30 \mathrm{~mm}$ in height.

\section{Instruments and Experiment Method}

Beryllium and stainless steel samples were polished mechanically then were pickled by $15 \% \mathrm{HCl}$ solution, and heating vacuum degassing. The diffusion bonding was achieved by hot pressing under 
the condition of heating temperature $750^{\circ} \mathrm{C}$ and diffusion persure $45 \mathrm{MPa}$ with holding time $2 \mathrm{~h}$.

The diffusion bonding samples for Be/00Cr17Ni14Mo2 stainless steel were ground wetly to No. 900 metallographic sandpaper then were polished mechanicaly. The adsorption and oxidation behavior for Be and Be/00Cr17Ni14Mo2 stainless steel welding joint were analysed by Auger electron spectroscopy (AES) and two secondary ion mass spectrometry (SIMS) in supper high vacuum atmosphere.

The base vacuum degree of AES vacuum chamber was less than $4 \times 10^{-8} \mathrm{~Pa}$. $\mathrm{Ar}+$ sputtering parameters were $4 \mathrm{kV}$ ion energy, $1 \times 1 \mathrm{~mm}^{2}$ sputtering scanning area, $2.4 \mu \mathrm{A}$ ion beam current, and adsorption after sputtering and cleaning samples. Auger electron spectroscopy parameters were $3 \mathrm{kV}$ electron excitation energy, $0.25 \%$ energy resolution, $100 \mathrm{nA}$ electron beam current. As sputtering, the vacuum degree is $2 \times 10^{-6} \mathrm{~Pa}$ due to $\mathrm{Ar}+$ into vacuum chamber.

\section{Results and Discussion}

The AES spectra of beryllium surface before and after Ar+ sputtering are shown in Fig. $1 \mathrm{a}$ and Fig. 1 b, respectively, which original beryllium surface before Ar+ sputtering contains large amounts of $\mathrm{C}$ and KLL auger peak linear show the performance characteristics of graphite, so it adsorbs mainly C. KLL auger peaks of beryllium surface before and after Ar+ sputtering locate at $95 \mathrm{eV}$ and $105 \mathrm{eV}$, which $95 \mathrm{eV}$ and $105 \mathrm{eV}$ are corresponding chemical states of $\mathrm{BeO}$ and $\mathrm{Be}$, respectively, so indicate that $\mathrm{Ar}+$ sputtering can clean beryllium surface.

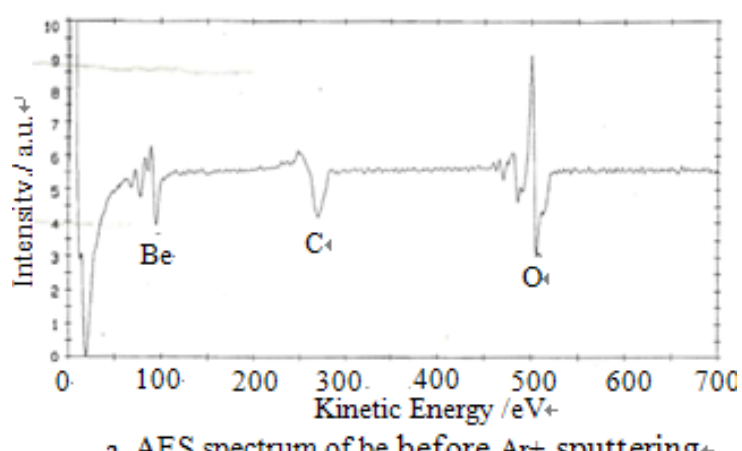

a. AES spectrum of be before Ar+ sputtering

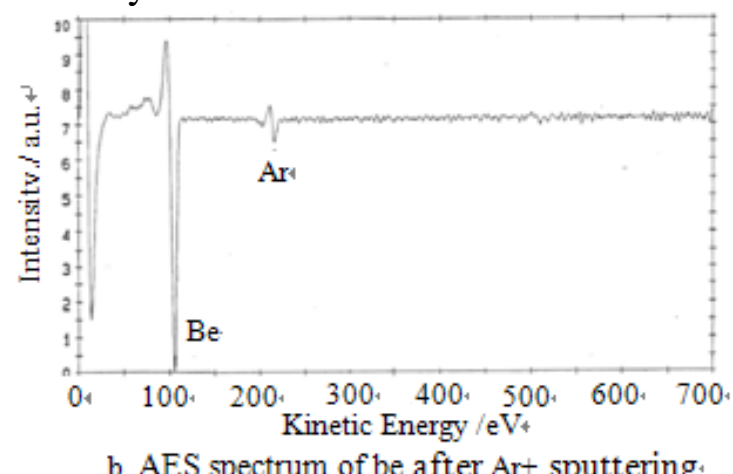

b. AES spectrum of be after Ar+ sputtering,

Fig. 1 AES spectra of Be surface before and after Ar+ sputtering

After Ar+ sputtering the surfaces of beryllium and 00Cr17Ni14Mo2 stainless steel, the contents of $\mathrm{C}$ and $\mathrm{O}$ at $4 \times 10^{-8} \mathrm{~Pa}$ and $5 \times 10^{-7} \mathrm{~Pa}$ atmosphere during beryllium surfaces adsorption and at $5 \times 10^{-7} \mathrm{~Pa}$ atmosphere during $00 \mathrm{Cr} 17 \mathrm{Ni} 14 \mathrm{Mo} 2$ stainless steel surfaces adsorption are shown in Fig. 2a, Fig. 2b and Fig. 2c, respectively, which line A is the place of beginning adsorption. Fig.2a shows that the increase of $\mathrm{C}$ and $\mathrm{O}$ atom is synchronization and its ratio is 1 during beryllium initial adsorption, and $\mathrm{O}$ and $\mathrm{C}$ atomic ratio is more than 1 after beryllium adsorbed 70 min in $4 \times 10^{-8} \mathrm{~Pa}$ atmosphere. At the same time, the $95 \mathrm{eV}$ auger peak formed and gradually strengthened but $\mathrm{C}$ peak

was almost no change during beryllium adsorption, which indicates that $\mathrm{C}$ always take adsorption state, and $\mathrm{O}$ and $\mathrm{C}$ atomic ratio is more than 1 due to occurred oxidation. Fig.2b shows that $\mathrm{C}$ adsorption strengthen and $\mathrm{O}$ adsorption is almost no change at $5 \times 10^{-7} \mathrm{~Pa}$ atmosphere during beryllium surfaces adsorption. Fig.2c shows that oxidation is strong but $\mathrm{C}$ adsorption is very weak at $5 \times 10^{-7} \mathrm{~Pa}$ atmosphere during SS surface adsorption. 


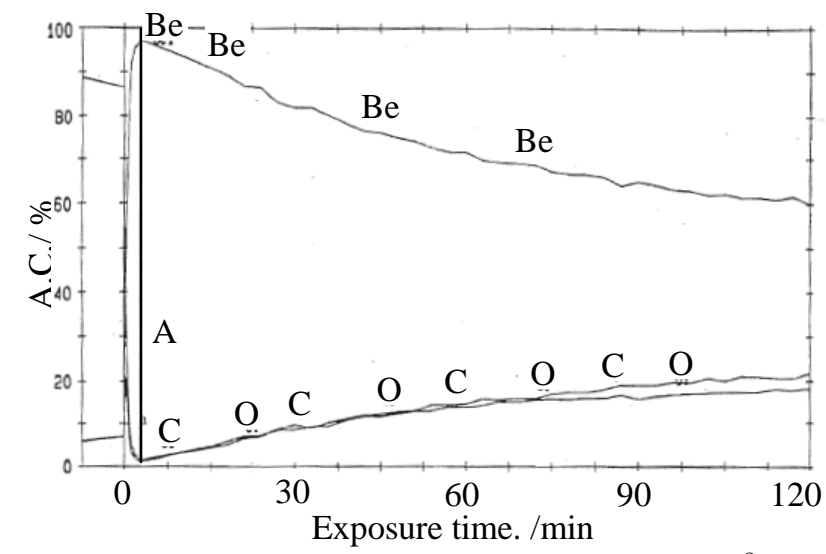

a. Contents of $\mathrm{C}$ and $\mathrm{O}$ on Be surface at $4 \times 10^{-8} \mathrm{~Pa}$

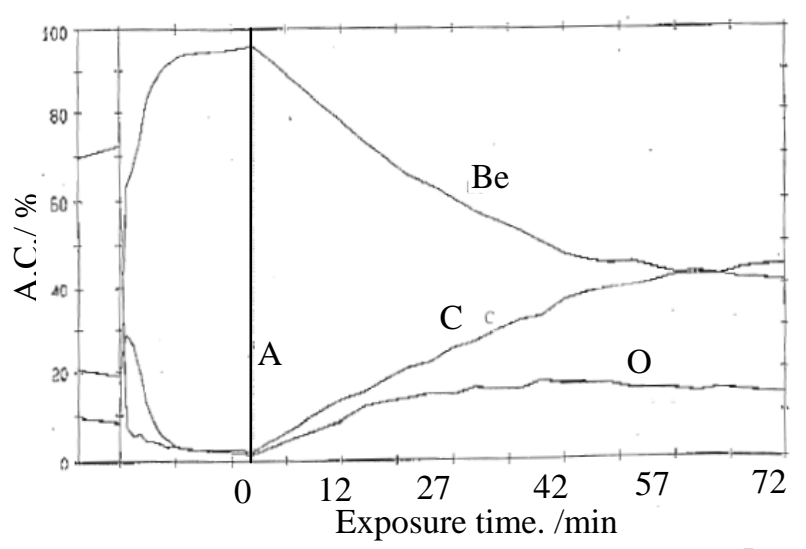

b. Contents of $\mathrm{C}$ and $\mathrm{O}$ on Be surface at $5 \times 10^{-7} \mathrm{~Pa}$

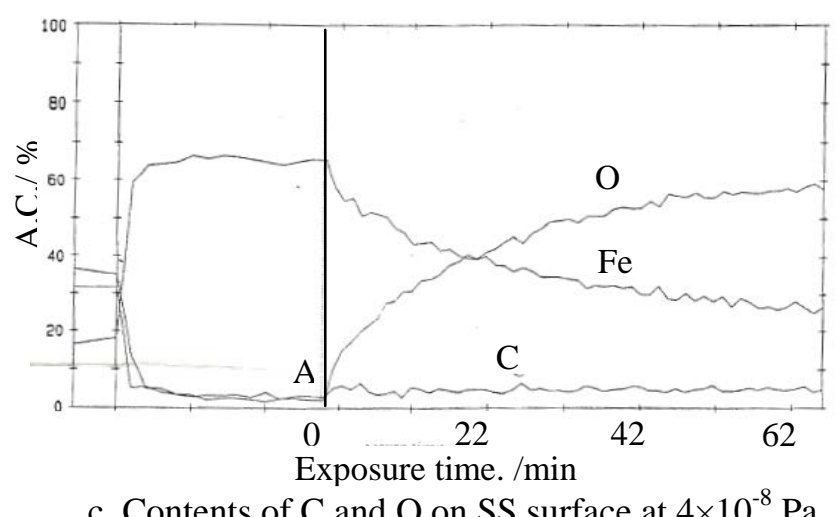

Fig. 2 Dependence of $\mathrm{C}$ and $\mathrm{O}$ contents on exposure

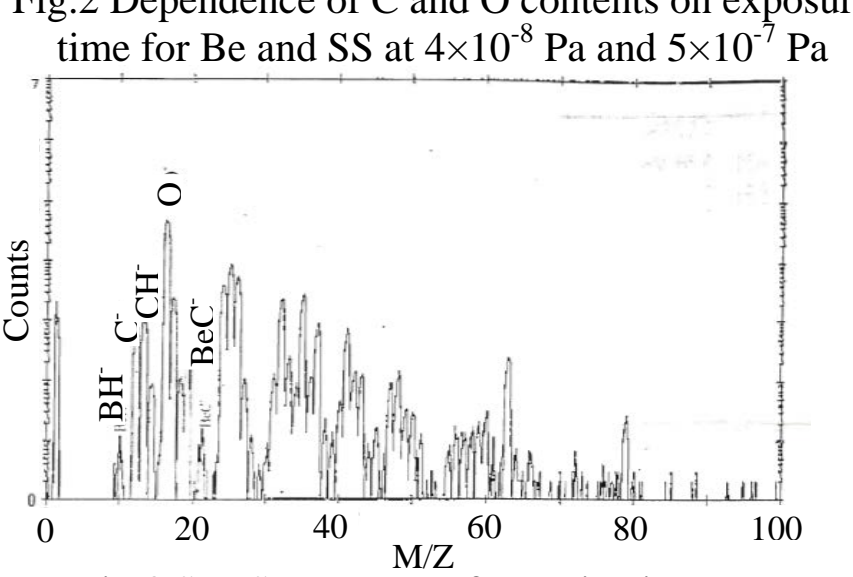

Fig.3 SIMS spectrum of negative ion on

Be surface exposed at $4 \times 10^{-8} \mathrm{~Pa}$ for $2 \mathrm{~h}$

The SIMS spectrum of negative ion for Be surface in supper high vacuum atmosphere is shown in Fig. 3, whose beryllium adsorption is mainly hydrocarbons with $\mathrm{CH}^{-}, \mathrm{BeO}^{-}, \mathrm{O}^{-}, \mathrm{BeH}^{-}, \mathrm{BeC}^{-}$, so it shows that beryllium excepted oxidation but also combined with $\mathrm{C}$ and $\mathrm{H}$. The beryllium adsorbed strongly in hydrocarbons, and its adsorption increased significantly with decrease in the vacuum degree and accompanied by oxidation which the oxidation degree was irrelevant with the vacuum degree in this experiment. Thus, the protective atmosphere, vacuum degree and welding surface cleanliness should been strictly controlled when beryllium welds with other material. The beryllium surface cleaning must be good before welding, otherwise formed compounds remain in the welding joint and form inclusion and segregation, which result in reducing welding performance. Use surface modification technology such as preparating various coating as a buffer layer or insulating layer on the beryllium surface[12][13] while Be welding to improve bonding strength.

The AES spectra of cleaned welding seam and SS matrix with Be exposed at filling $\mathrm{Ar}+$ to $4 \times 10^{-6} \mathrm{~Pa}$ (the base vacuum degree is $3 \times 10^{-8} \mathrm{~Pa}$ in vacuum chamber.) for $2 \mathrm{~h}$ are shown in Fig. $4 \mathrm{a}$ 
and Fig. 4 b, respectively, which compared SS or other metal, the welding seam and SS matrix with Be adsorbed strongly in $\mathrm{C}$ and oxidated seriously, and the more beryllium content of the welding seam and SS matrix, the more strong oxidation ability. The welding seam and SS matrix with Be are similar to strong adsorption of beryllium metal. The reason is that Be diffused into SS matrix and reduced its surface adsorption energy. The C KLL auger peak linear shows that adsorbed mainly graphite, and Be KLL auger peaks of the welding seam locate at $95 \mathrm{eV}$, which has formed the $\mathrm{BeO}$, while Be KLL auger peaks of the SS matrix with Be locate at $105 \mathrm{eV}$. So pretreatment of beryllium surface and control of bonding atmosphere are important effect on diffusion bonding.

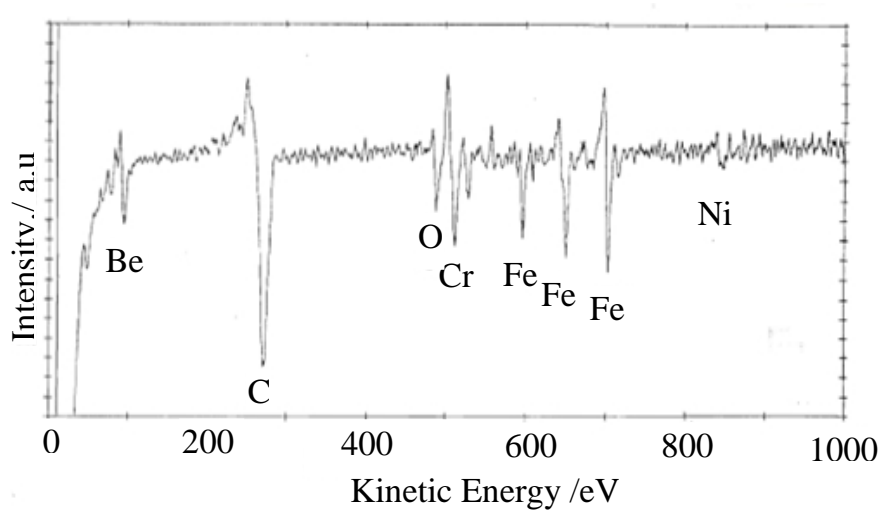

a. AES spectrum of cleaned welding seam

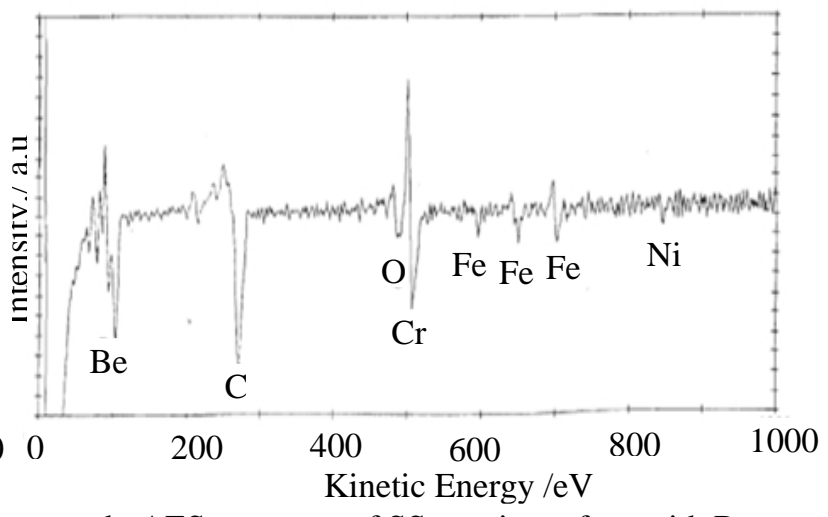

b. AES spectrum of SS matrix surface with Be

Fig.4 AES spectra of cleaned welding seam and SS matrix with Be exposed at $3 \times 10^{-8} \mathrm{~Pa}$ for $2 \mathrm{~h}$

\section{Conclusion}

Beryllium, welding seam and 00Cr17Ni14Mo2 stainless steel matrix with beryllium adsorbed strongly in $\mathrm{C}$ and $\mathrm{O}$ even in super high-vacuum atmosphere, and accompanied by serious oxidation. The beryllium adsorption decreased its surface cleanliness and formed oxides, which compounds remained in the welding joint and formed inclusion and segregation, and resulted in reducing bonding strength. Thus, pretreatment of beryllium surface and control of bonding atmosphere are significantly important, which can determine directly the success or failure of diffusion bonding for Be/00Cr17Ni14Mo2 stainless steel, and there is realistic significance for beryllium welding with other material. The magnetron sputtering ion coating method can ensure that the surface cleaning of beryllium and 00Cr17Ni14Mo2 stainless steel, and eliminate the adverse effects caused by the surface oxidation.

\section{References}

[1] Allouche A.,Ferro Y.. First-Principles Study of hydrogen retention and diffusion in beryllium oxide [J]. 2015:272:91-100.

[2] Roth J.,Wampler W.R.,Oberkofler M., ET al. Deuterium retention and out-gassing from beryllium oxide on beryllium [J]. Journal of Nuclear Materials. 2014:453 (1-3):27-30.

[3] Roth J.,Doerner R.,Baldwin M., ET al. Oxidation of beryllium and exposure of beryllium oxide to deuterium plasmas in PISCES B [J]. Journal of Nuclear Materials. 2013:438:S1044-S1047.

[4] Allouche A.. Nitrogen reactivity toward beryllium: surface reactions [J]. Journal of PhysicsCondensed Matter. 2013:25 (22).

[5] Zhang Yulong, Zhang Weifang, Zhang Aiai, ET al. Study on Oxidation Behavior of Beryllium Bronze [J]. Advanced Materials Research. 2013:750-752:683-686.

[6] Allouche A.. Carbon monoxide adsorption on beryllium surfaces [J]. Surface Science. 2012: 608:265-274.

[7] Jahn M.,Mueller M.,Endlich M., ET al. Oxygen vibrations and acoustic surface plasmon on 
Be(0001) [J]. Physical Review B. 2012:86 (8).

[8] Allouche A.. Quantum Modeling of Water and Oxygen Adsorption on Beryllium Surface [J]. Journal of Physical Chemistry C. 2012:116 (7):4662-4670.

[9] Allouche A.. Quantum Modeling of Beryllium Surface Oxidation and Hydrogen Adsorption [J]. Journal of Physical Chemistry C. 2011:115 (16):8233-8239.

[10] Mallinson Christopher F.,Castle James E.,Watts John F.. The electron spectra of beryllium and beryllium oxide: an XPS, X-AES and AES study [J]. Surface and Interface Analysis. 2014:46 (11-12):989-992.

[11] Ferro Yves,Allouche Alain,Linsmeier Christian. Absorption and diffusion of beryllium in graphite, beryllium carbide formation investigated by density functional theory [J]. Journal of Applied Physics.2013:113 (21).

[12] Kablov E.N.,Solntsev S.S.,Rozenenkova V.A., ET al. Composite glass-metal coatings for protecting beryllium at high temperatures [J]. Glass and Ceramics.2012:69:113-116.

[13] Rupprecht M,Wolfrum C,Pfammatter D, ET al. Plate-like zinc magnesium alloy pigments, useful in a corrosion protective coating of aircraft or in coil coatings, comprise zinc, magnesium, manganese, beryllium, yttrium, lithium and/or tin, and aluminum, titanium, iron and/or copper. Patent Number(s):DE102012107633-A1,WO2014029781-A2,WO2014029781-A3,EP2785806-A2: 2014. 